\title{
A Congenital Deadly Association: Dilated Cardiomyopathy and Long QT Syndrome
}

Neiberg de Alcantara Lima ${ }^{1, *}$, Antonio Thomaz de Andrade ${ }^{2}$, Stela MV Sampaio ${ }^{2}$, Mark Loehrke ${ }^{1}$

\section{ORCID IDs}

Lima NA (D) https://orcid.org/0000-0003-3616-435X

Andrade AT (D) https://orcid.org/0000-0002-1494-8981

Sampaio SMV (D) https://orcid.org/0000-0003-1314-2107

Loehrke M (D) https://orcid.org/0000-0002-9681-2999

\begin{abstract}
Long QT syndrome is one of the most feared entities in hospitalized patients due to the potential risk for ventricular tachycardia and sudden death. Association between channelopathies and congenital cardiomyopathy is a new entity that has been studied recently. We report an interesting case of this association that maybe related to a genetic mutation.
\end{abstract}

KEYWORDS: Long QT Syndrome; Cardiomyopathies; Genetic diseases; Ventricular tachycardia; Channelopathies.

1. Western Michigan University - Homer Stryker M.D. School of Medicine - Department of Internal Medicine - Kalamazoo/MI - USA. 2. Hospital de Messejana - Fortaleza/CE - Brazil. 


\section{LEARNING OBJECTIVE}

Congenital long QT syndrome and congenital cardiomyopathy can be both associated to SCN5A mutations. Despite the fact that this is a rare association, it is important for physicians to be aware of it due to the potential high risk of ventricular arrythmias and sudden death, especially in young patients.

\section{INTRODUCTION}

Long QT syndrome (LQTS) is one of the most feared entities in hospitalized patients due to the potential risk for ventricular tachycardia (VT) and sudden death, happening sometimes in completely asymptomatic patients. Clinicians are usually cautious with QT interval prolongation as many common hospital drugs and electrolytes abnormalities can prolong QT and trigger $\mathrm{VT}^{1}$.

Since described in 1957, LQTS has been intensively studied, but the association between channelopathies and congenital cardiomyopathy is a relatively new association ${ }^{2}$. This report presents an interesting case with this association and correlates with the current medical literature.

\section{CASE REPORT}

A 45-year-old man with a medical history of mild hypertension presented with progressive dyspnea and anasarca. Family history was not contributory.Jugular venous distension, pedal edema and pulmonary rales were observed. No history of alcohol or drug abuse.

Electrocardiogram (EKG) revealed sinus rhythm, left ventricular hypertrophy and a QTc of $440 \mathrm{~ms}$ (Fig. 1a). Echocardiogram showed severe left atrial and ventricular dilatation with an ejection fraction of $20 \%$. Started on guideline-based therapy for heart failure with bisoprolol, furosemide, losartan and spironolactone.

At the 5th day of hospitalization, he was diagnosed with a respiratory infection and moxifloxacin was initiated. At the 8th day, he had an episode of ventricular fibrillation followed by two episodes of pulseless polymorphic ventricular tachycardia and monomorphic ventricular tachycardia. Post arrest EKG showed QTc of $550 \mathrm{~ms}$ (Fig. 1b).

The antibiotic was discontinued and potassium and magnesium were replaced. Coronary arteries had no lesions. Patient discharged for outpatient follow up after stabilization with a diagnosis of iatrogenic acquired LQTS. Electrocardiogram at discharge QTc was 450 ms (Fig. 1c).

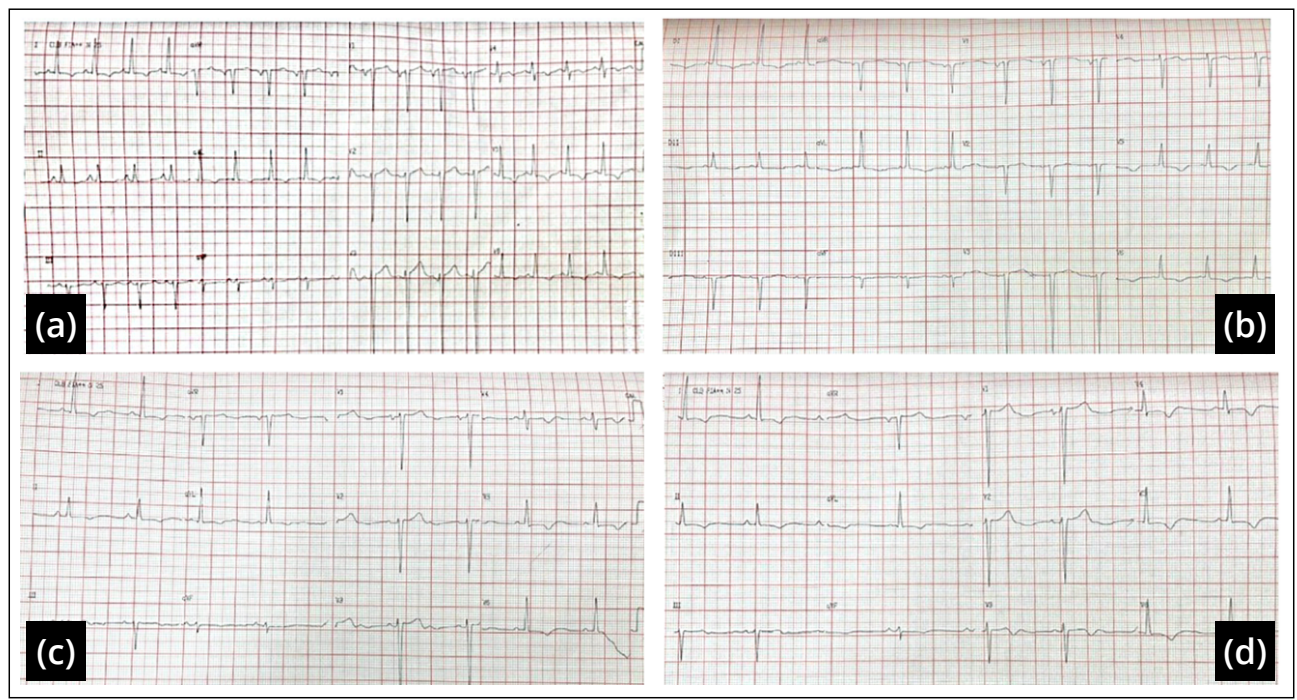

Figure 1. Twelve-lead electrocardiogram showing different QT intervals. (a) admission QTc 440 ms. (b) after cardiac arrest, QTc $550 \mathrm{~ms}$. (c) at the discharge $450 \mathrm{~ms}$. (d) one month after discharge $440 \mathrm{~ms}$. 
A month later QTc was $440 \mathrm{~ms}$ off QT prolonging drugs (Fig. 1d), but a 24-hour Holter showed intermittent QT prolongation up to $530 \mathrm{~ms}$ (Fig. 2). Treadmill test showed postexercise complex polymorphic ventricular tachycardia associated with QT prolongation up to $506 \mathrm{~ms}$ (Fig. 3). Findings consistent with type 3 long QT syndrome (LQTS-3) associated with dilated cardiomyopathy may related to SCN5 A mutation.

Patient declined genetic testing. An implantable cardioverter deffibrilator (ICD) was placed and he has been New York Heart Association (NYHA) class II on oral guideline-based heart failure medications.

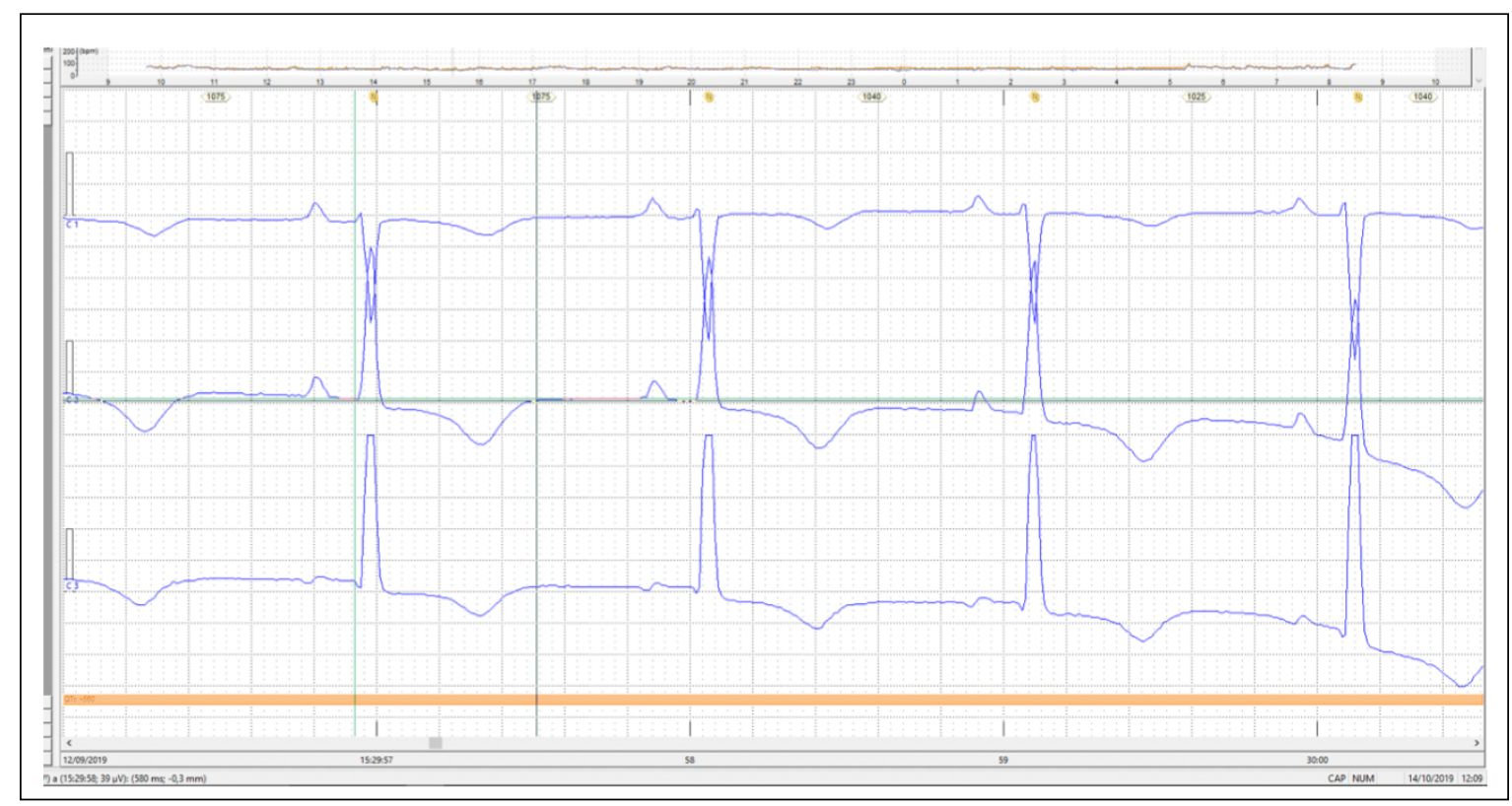

Figure 2. 24-hour Holter showing QTc at 530 ms.

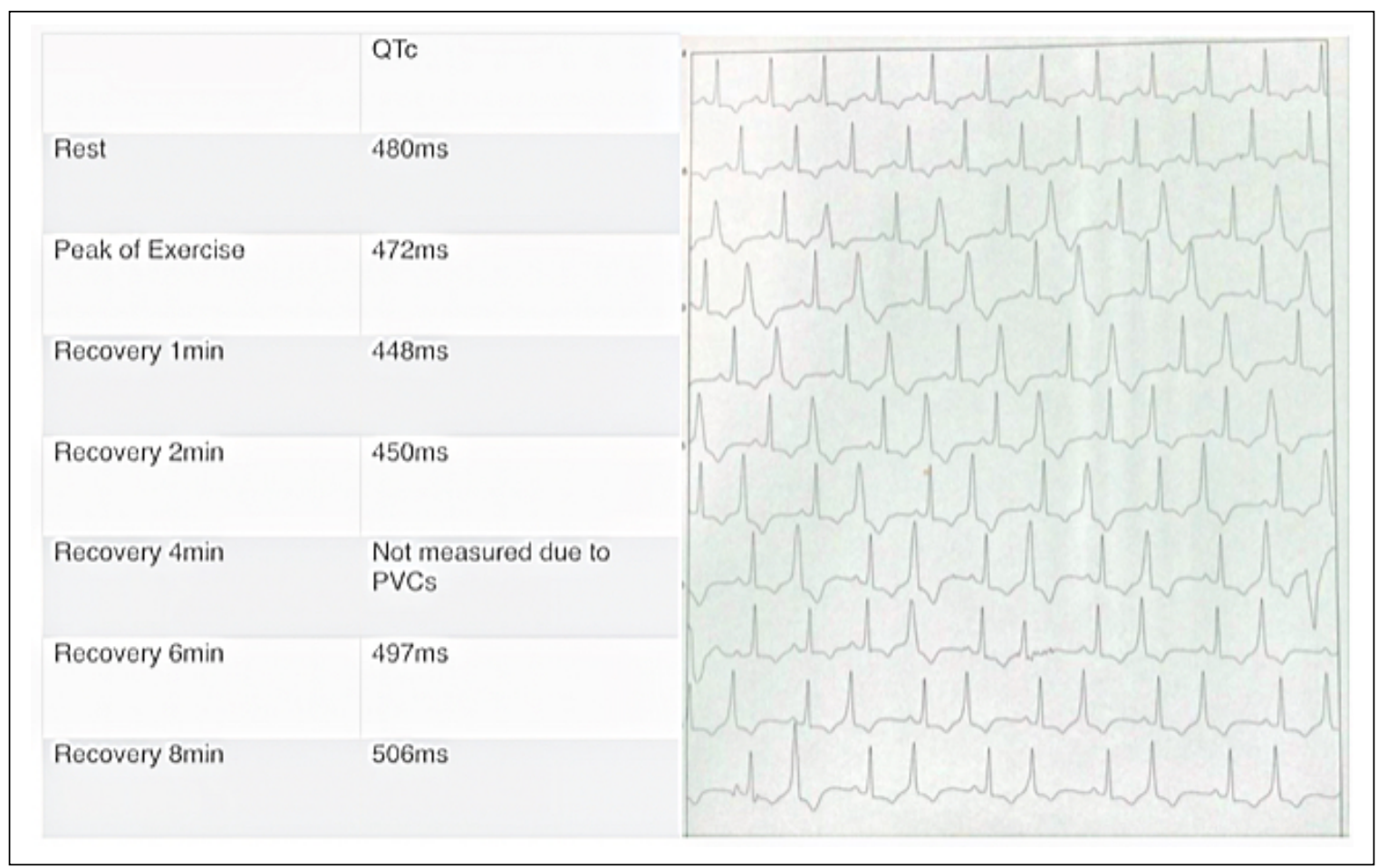

Figure 3. Stress test. Left - QTc by time. Right - polymorphic premature ventricular contractions during recovery time. 


\section{DISCUSSION}

Acquired LQTS is by far more prevalent than congenital LQTS; however, in the presence of congenital channelopathies, electrolytes abnormalities and drugs can more easily prolong the QT interval and trigger ventricular arrhythmias ${ }^{1}$. In this patient, QT interval improved after the discontinuation of the antibiotic, he was initially misdiagnosed with acquired LQTS. Subsequent follow up in the absence of QT prolonging agents showed the presence of intermittent prolonged QT interval.

It is widely known the QT interval can vary due to heart rate, electrolyte level and autonomic changes. An isolated 12-leads EKG might be inappropriate to evaluate patients with LQTS, especially type 2 and $3^{3,4}$. In the described patient, a 24-hour Holter after one month showed prolonged QT intervals that were not seen at the discharge. Different strategies to measure the QT variability on 24-hour Holter in LQTS patients have been recently published and should be used in the suspicion for LQTS ${ }^{3,4}$.

Schwartz's criteria are a widely accepted tool for the diagnosis of congenital LQTS, and the studied patient scored 5 points. It is necessary > 3.5 points to have high probability of LQTS ${ }^{4}$. There are more than 10 different subtypes of congenital LQTS even though genetic analysis is necessary to determine the subtype, EKG changes on treadmill might contribute to the diagnosis. During the peak of exercise QT interval decreases, but during the recovery phase it increases in patients with LQTS type 2 and $3^{5,6}$. The EKG also suggests LQTS-3 due to long isoelectric ST segment.

Type 3 long QT syndrome patients present more often with bradycardia, and QT prolongation is more pronounced during slow rate therefore they have more arrhythmic events at rest ${ }^{7}$.

The mutation more associated with LQTS-3 occurs in the SCN5A gene. This gene encodes the pore forming ion-conductiong a-subunit of the cardiac sodium channel, which is responsible for the initiation and propagation of action potentials and thereby determines excitability and conduction of the electrical stimuli through the heart. Usually in a patient with LQTS-3, there is a gain of function leading to a pathological increase of sodium influx into cardiomyocytes through aberrant channel gating and causes LQTS ${ }^{7}$.

SCN5A mutations are also associated to other pathologies such as Brugada syndrome and cardiomyopathy. The patient did not have any classical risk factors for heart failure therefore in the setting of LQTS-3 and possible SCN5A mutation the etiology of the cardiomyopathy might be genetic ${ }^{2}$.

SCN5A mutations can cause a proton leak into the cardiomyocyte through the Sodium channel, or increased $\mathrm{Na}+$ influx caused by gain-of-function variants, may lead to compensatory activation of the $\mathrm{N}^{+} / \mathrm{H}^{+}$or $\mathrm{the}^{\mathrm{N}} \mathrm{Ca}^{+} / \mathrm{Ca}^{2+}$ exchanger, thus leading to intracellular acidification or calcium overload, respectively, and consequent impaired excitation-contraction coupling and/or myocardial damage with subsequent heart failure ${ }^{2,8,9}$.

American Heart Association guidelines recommend ICD implantation for patients with LQTS-3 especially in higher risk patients, therefore the ICD was placed ${ }^{10}$. He also would meet criteria for ICD placement for being a sudden death survivor in the setting of severe cardiomyopathy ${ }^{10}$.

Even though the presented patient refused genetic testing, all clinical and electrocardiographic evidence suggests he has a SCN5A mutation with associated LQTS-3 and cardiomyopathy.

Despite the fact that this is a rare association, it is important for physicians to be aware of it due to the potential high risk of ventricular arrythmias and sudden death, especially in young patients.

\section{AUTHOR'S CONTRIBUTION}

Conceptualization, Lima NA, Andrade AT and Sampaio SMV; Methodology, Lima NA, Andrade AT and Sampaio SMV; Writing - Original Draft; Lima NA, Andrade AT and Sampaio SMV; Writing - Review and Editing; Sampaio SMV and Loehrke M; Supervision, Sampaio SMV and Loehrke M. 


\section{REFERENCES}

1. El-Sherif N, Turitto G, Boutjdir M. Acquired long QT syndrome and torsade de pointes. Pacing Clin Electrophysiol. 2018;41(4):414-21. https://doi.org/10.1111/pace.13296

2. Asatryan B. Cardiac sodium channel dysfunction and dilated cardiomyopathy: a contemporary reappraisal of pathophysiological concepts. J Clin Med. 2019;8(7):1029. https://doi.org/10.3390/jcm8071029

3. Ogawa Y, Tanaka T, Kido S. Maximum QTc on Holter electrocardiography in children. Pediatr Int. 2018;60(6):507-12. https://doi. org/10.1111/ped.13556

4. Robyns T, Willems R, Vandenberk B, Ector J, Garweg C, Kuiperi C, et al. Individualized corrected QT interval is superior to QT interval corrected using the Bazett formula in predicting mutation carriage in families with long QT syndrome. Heart Rhythm. 2017;14(3):376-82. https://doi.org/10.1016/j.hrthm.2016.11.034

5. Schwartz PJ, Crotti L. QTc Behavior during exercise and genetic testing for the long-QT syndrome. Circulation. 2011;124:2181-4. https://doi.org/10.1161/CIRCULATIONAHA.111.062182

6. Horner JM, Horner MM, Ackerman MJ. The diagnostic utility of recovery phase QTc during treadmill exercise stress testing in the evaluation of long QT syndrome. Heart Rhythm. 2011;8(11):1698-704. https://doi.org/10.1016/j.hrthm.2011.05.018

7. Wilde AAM, Amin AS. Clinical spectrum of SCN5A mutations: Long QT syndrome, Brugada syndrome, and cardiomyopathy. JACC Clin Electrophysiol. 2018;4(5):569-79. https://doi.org/10.1016/j.jacep.2018.03.006

8. Moreau A, Gosselin-Badaroudine P, Boutjdir M, Chahine M. Mutations in the voltage Sensors of Domains I and II of Nav1. 5 that are associated with arrhythmias and dilated cardiomyopathy generate gating pore currents. Front Pharmacol. 2015;6:301. https://doi. org/10.1016/j.hrthm.2014.05.031

9. Amin AS. SCN5A-related dilated cardiomyopathy: What do we know? Heart Rhythm 2014;11(8):1454-5. https://doi.org/10.1016/j. hrthm.2014.05.031

10. Al-Khatib SM, Stevenson WG, Ackerman MJ, Bryant WJ, Callans DJ, Curtis AB, et al. 2017 AHA/ACC/HRS Guideline for management of patients with ventricular arrhythmias and the prevention of sudden cardiac death: A report of the American College of Cardiology/American Heart Association Task Force on Clinical Practice Guidelines and the Heart Rhythm Society. J Am Coll Cardiol. 2018;72(14):e91-220. https://doi.org/10.1016/j.jacc.2017.10.054 